\title{
Cardiac magnetic resonance characteristics of acute myocarditis occurring after mRNA-based COVID-19 vaccines immunization
}

\author{
Jaroslaw Meyer-Szary ${ }^{1}$, Magdalena Bazgier ${ }^{1} \oplus$, Paulina Lubocka ${ }^{1} \oplus$, \\ Karolina Dorniak $^{2} \odot$, Robert Sabiniewicz ${ }^{1} \odot$ \\ ${ }^{1}$ Deptartment of Pediatric Cardiology and Congenital Heart Diseases, Faculty of Medicine, \\ Medical University of Gdansk, Poland \\ ${ }^{2}$ Department of Cardiac Diagnostics, Faculty of Medicine, Medical University of Gdansk, Poland
}

Almost 1 billon people worldwide have been fully vaccinated. Recent publications report on several cases of presumably vaccine-induced myocarditis [1-3]. This article presents a series of patients with this condition.

Case 1. A 29-year-old male presented with severe chest tightness, dyspnea and retrosternal pain radiating to the left arm 2 days after receiving Spikevax Vaccine Moderna. Resting electrocardiogram (ECG) revealed pattern typical for cardiac muscle injury, alongside with increased inflammation parameters (peak C-reactive protein [CRP] of $39 \mathrm{mg} / \mathrm{L}$, peak troponin I above $50 \mathrm{ng} / \mathrm{mL}$ ). Echocardiography revealed normal cardiac function with left ventricular ejection fraction (LVEF) of $65 \%$ and tricuspid annular plane systolic excursion (TAPSE) of $23 \mathrm{~mm}$. Acute coronary syndrome and pulmonary embolism were excluded with coronary angiography and computed tomography, respectively. On the $7^{\text {th }}$ day after vaccination, cardiac magnetic resonance (CMR) was performed, revealing LVEF of $62 \%$ and signs of edema and acute muscle injury in inferior, infero-lateral and antero-lateral segments. Subepicardial/intramural late gadolinium enhancement (LGE) was also found in the same regions, corresponding to the extent of edema. No viral diagnostics were performed.

Case 2. A 12-year-old male presented with severe stabbing chest pain, diarrhea and fever 2 days after receiving Comirnaty vaccine. Resting ECG revealed a pattern typical for cardiac muscle injury (ST elevation in leads I, II, V3-V6), alongside with increased inflammation parameters (peak CRP of $23 \mathrm{mg} / \mathrm{L}$, peak troponin I of $5.1 \mathrm{ng} / \mathrm{mL}$ ). On the $3^{\text {rd }}$ day after vaccination LVEF of $58 \%$ and TAPSE of $18 \mathrm{~mm}$ were noted in echocardiography. On the $4^{\text {th }}$ day, CMR was performed, revealing EF of $56 \%$ and subepicardial/intramural LGE in the basal infero-lateral and basal inferior wall. In extended viral diagnostics with polymerase chain reaction (PCR) and serology methods no infectious cause of myocarditis was found. No lymphocyte populations abnormalities were found. The patient required only analgesic treatment (paracetamol) during $1^{\text {st }}$ day of hospitalization. Day 5 evaluation showed improved systolic function (LVEF $72 \%$, TAPSE $25 \mathrm{~mm}$ ).

Case 3. A 17-year-old male presented with severe stabbing chest pain and fever 1 day after receiving Comirnaty vaccine. Resting ECG revealed pattern typical for cardiac muscle injury (ST elevation in leads II, III, aVF), alongside with increased inflammation parameters (peak CRP of $18.2 \mathrm{mg} / \mathrm{L}$, peak troponin I $8.9 \mathrm{ng} / \mathrm{mL}$ ). In echocardiography on the $2^{\text {nd }}$ day after vaccination LVEF of $63 \%$ and TAPSE of $26 \mathrm{~mm}$ were described. In the follow-up ECG evaluation T waves inversion in leads II, III, aVF, V6 was observed. On the $6^{\text {th }}$ day CMR was performed, revealing EF of $60 \%$ and subepicardial features of edema/acute injury as well as LGE areas in the basal and mid-ventricular infero-lateral and inferior segments. In extended viral diagnostics

Address for correspondence: Jarosław Meyer-Szary, MD, PhD, Department of Pediatric Cardiology and Congenital Heart Diseases, Faculty of Medicine, Medical University of Gdansk, ul. M. Skłodowskiej-Curie 3a, 80-210 Gdańsk, Poland, e-mail: jmeyerszary@gumed.edu.pl

Received: 31.07.2021 Accepted: 24.10.2021 Early publication date: 15.11.2021

This article is available in open access under Creative Common Attribution-Non-Commercial-No Derivatives 4.0 International (CC BY-NC-ND 4.0) license, allowing to download articles and share them with others as long as they credit the authors and the publisher, but without permission to change them in any way or use them commercially. 


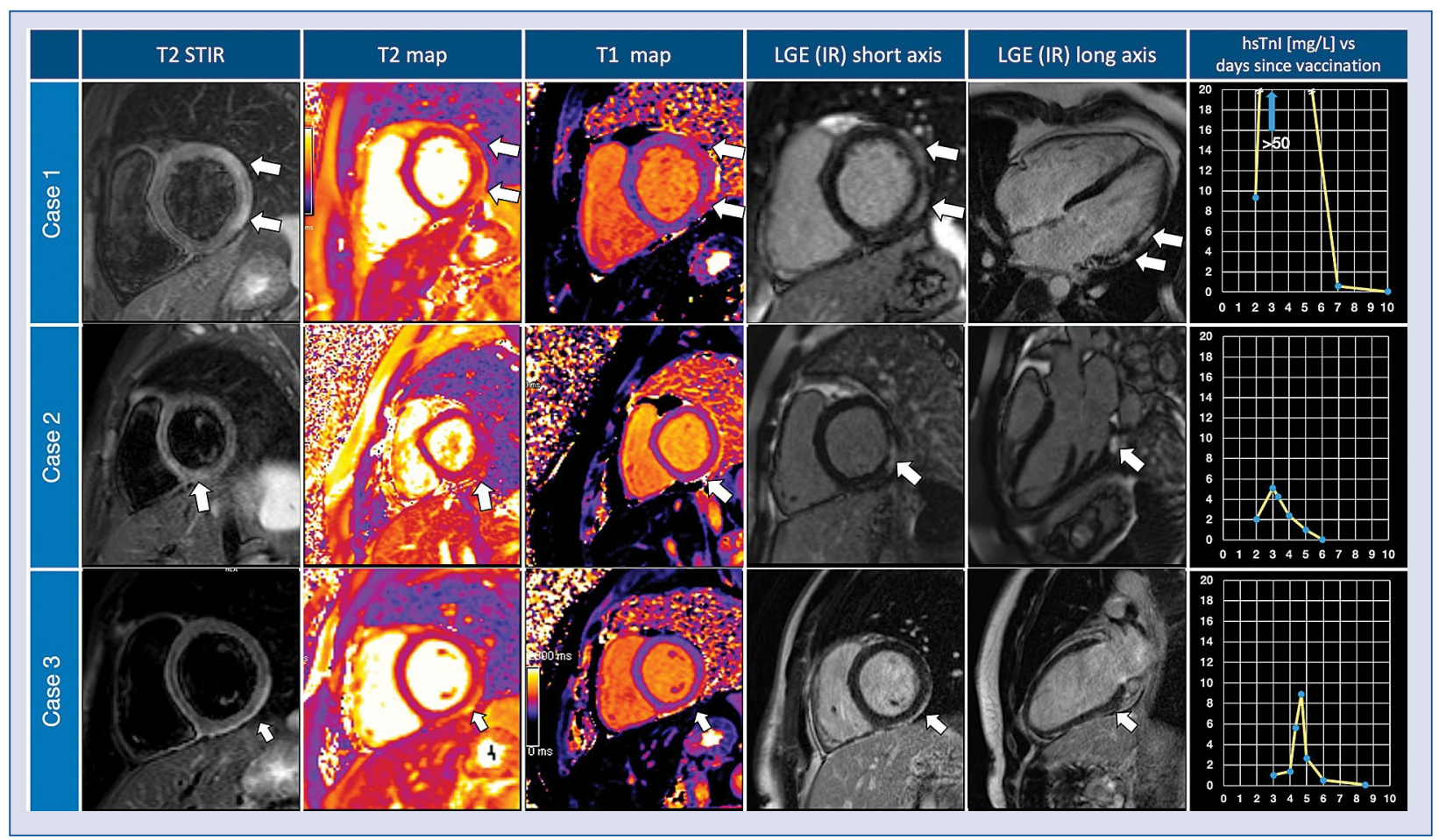

Figure 1. Case 1. Subepicardial hyperintensity consistent with edema (i.e., features of acute injury) in the basal inferior, infero-lateral and antero-lateral segments in the T2-weighted short tau inversion recovery (STIR) image, paralleled by increased T2 and T1 relaxation times in parametric mapping and a matching late gadolinium enhancement (LGE) area suggestive of irreversible damage (arrows); Case 2. Mild localized edema found in the basal inferior and infero-lateral segments in the T2 STIR image, again paralleled by increased T2 and T1 relaxation times and a small LGE patch found solely in the basal infero-lateral segment (arrows); Case 3. Mild subepicardial edema in T2 STIR closely matched by T1 and T2 increase in parametric mapping, as well as subtle subepicardial LGE in the basal inferior and infero-lateral segments (arrows); IR — inversion recovery; hsTnl — high sensitivity troponin I plasma levels by days since vaccination.

with PCR and serology methods, no infectious agents were found. Lymphocyte populations were normal.

The current patients have several common characteristics. All three were young males and had no comorbidities. In all three, myocarditis developed shortly after the $2^{\text {nd }}$ dose of mRNA vaccine against coronavirus disease 2019 (COVID-19), within 1 to 5 days. None of the patients had a history of previous COVID-19 disease. All of them presented with elevated troponin levels, peaking on the $3^{\text {rd }}$ or $4^{\text {th }}$ day after vaccination. Apart from the troponin, CK-MB mass, liver enzymes and CRP were elevated in all patients. No other laboratory abnormalities were found. On echocardiography the LVEF was within the normal range in all of these cases and no regional wall motion abnormalities were found. Interestingly, in all three subjects CMR revealed a similar pattern of myocardial injury found predominantly in the inferior and inferolateral segments (Fig. 1). This pattern included localized features of subepicardial edema in T2-weighted short tau inversion recovery (STIR) images, accompanied by elevated native T1 and $\mathrm{T} 2$ relaxation times in cardiac parametric mapping, with corresponding foci of LGE (i.e., areas of predominantly irreversible myocardial injury) in the respective regions. The clinical course was favorable in all three cases. They only transiently received analgesia (paracetamol). The hospital course ranged from 5 to 9 days $(9,5,5$, respectively). All had normalization of ECG and laboratory parameters, were asymptomatic and in good general condition on discharge.

Noticeably, no similar cases were reported in the course of registration trials of any of the vaccines $[4,5]$. This might be due to the low incidence of this adverse effect. So far, in the documented region, with over 450,000 fully vaccinated inhabitants, only $3(0.0007 \%)$ cases of vaccine-induced myocarditis have been identified. Similarly, in Israel, the development of myocarditis within 30 days from the vaccination was reported in 121 subjects per over 5 million fully vaccinated 
citizens $(0.002 \%)$. The present series includes only male patients, concordantly with the published reports also showing male predominance $[2,6]$.

The causal relation between myocarditis and COVID-19 vaccination has not been proven. However, the time concordance is quite indicative. Also, the mechanism responsible for triggering this adverse reaction has not been explained. The subjects with post-vaccine myocarditis do not present abnormally elevated anti-COVID-19 antibodies nor other laboratory markers that could differentiate them from patients suffering from viral myocarditis $[2,7,8]$. The current findings are consistent with previous reports.

The severe acute respiratory syndrome coronavirus 2 (SARS-CoV-2) can cause damage to the myocardium in two possible ways: primarily, by direct myocyte injury after binding to angiotensin-converting enzyme 2 (ACE-2) receptor [2], but also in the late phase of infection, in the process of cytokine storm [9]. Given that the post-vaccine myocarditis occurs predominantly after the second dose of mRNA specimen, which causes greater lymphocyte activation [10], it is more likely to be immune-mediated than related directly to the injected substance, however the precise pathway remains unknown. Contrary to a previous report [7] we found normal lymphocyte distributions in one and mildly elevated NK level in the second one (the first one was not assessed).

At present, unquestionable benefits of anti-COVID-19 vaccines outweigh the low risk of developing myocarditis that is mild and transient and should not be considered a reason to withhold vaccine administration. This has been clearly stated by the Advisory Committee on Immunization Practices [10]. Further research is necessary to investigate the pathomechanism of this adverse reaction, identify subjects at risk and implement adequate means of prevention.

Conflict of interest: None declared

\section{References}

1. Habib MB, Hamamyh T, Elyas A, et al. Acute myocarditis following administration of BNT162b2 vaccine. IDCases. 2021; 25: e01197, doi: 10.1016/j.idcr.2021.e01197, indexed in Pubmed: 34189042.

2. Deb A, Abdelmalek J, Iwuji K, et al. Acute myocardial injury following COVID-19 vaccination: a case report and review of current evidence from vaccine adverse events reporting system database. J Prim Care Community Health. 2021; 12: 21501327211029230, doi: 10.1177/21501327211029230, indexed in Pubmed: 34219532.

3. Watkins K, Griffin G, Septaric K, et al. Myocarditis after BNT162b2 vaccination in a healthy male. Am J Emerg Med. 2021 [Epub ahead of print], doi: 10.1016/j.ajem.2021.06.051, indexed in Pubmed: 34229940.

4. Baden LR, El Sahly HM, Essink B, et al. COVE Study Group. Efficacy and Safety of the mRNA-1273 SARS-CoV-2 Vaccine. N Engl J Med. 2021; 384(5): 403-416, doi: 10.1056/NEJMoa2035389, indexed in Pubmed: 33378609.

5. Polack FP, Thomas SJ, Kitchin N, et al. C4591001 Clinical Trial Group. Safety and Efficacy of the BNT162b2 mRNA Covid-19 Vaccine. N Engl J Med. 2020; 383(27): 2603-2615, doi: 10.1056/ NEJMoa2034577, indexed in Pubmed: 33301246.

6. Maron B, Udelson J, Bonow R, et al. Eligibility and disqualification recommendations for competitive athletes with cardiovascular abnormalities: Task Force 3: hypertrophic cardiomyopathy, arrhythmogenic right ventricular cardiomyopathy and other cardiomyopathies, and myocarditis. Circulation. 2015; 132(22), doi: 10.1161/cir.0000000000000239.

7. Muthukumar A, Narasimhan M, Li QZ, et al. In-depth evaluation of a case of presumed myocarditis after the second dose of COVID-19 mRNA vaccine. Circulation. 2021; 144(6): 487-498, doi: 10.1161/CIRCULATIONAHA.121.056038, indexed in Pubmed: 34133883.

8. Shay DK, Shimabukuro TT, DeStefano F. Myocarditis occurring after immunization with mRNA-Based COVID-19 vaccines. JAMA Cardiol. 2021; 6(10): 1115-1117, doi: 10.1001/jamacardio.2021.2821, indexed in Pubmed: 34185047.

9. Bajaj R, Sinclair H, Patel K, et al. Delayed-onset myocarditis following COVID-19. Lancet Resp Med. 2021; 9(4): e32-e34, doi: 10.1016/s2213-2600(21)00085-0.

10. Pfizer-BioNTech. Vaccines and Related Biological Products Advisory Committee Meeting FDA Briefing Document Pfizer-BioNTech COVID-19 Vaccine Sponsor: Pfizer and BioNTech. U.S. Food and Drug Administration. https://www.fda.gov/media/144245/download (Accessed 31.07.2021). 